\title{
ERRATUM
}

\section{A way forward along domain walls}

Hélène Béa and Patrycja Paruch

Nature Materials 8, 168-169 (2009); published online: 20 February 2009; corrected after print: 20 February 2009.

In the print version of this News \& Views article the reference list should have included the following:

3. Spaldin, N. A. \& Fiebig, M. Science 309, 391-392 (2005).

This reference is included in the HTML and PDF versions. 